\title{
THE SINUSOIDAL CRACK
}

\author{
XIAOPING LU and MARIA COMNINOU \\ Department of Mechanical Engineering and Applied Mechanics, The University of Michigan, \\ Ann Arbor, MI 48109-2125, U.S.A.
}

\begin{abstract}
The effects of mixed mode and partial crack closure due to curvature are studied by a simple sinusoidal crack model. For a slightly curved crack under applied shear, a small mode I stress intensity factor is obtained. Combined loads are also considered.
\end{abstract}

\section{INTRODUCTION}

IF AN INFINITE plane containing a plane crack is subjected to pure shear at its distant boundaries, the crack does not tend to open or close. Instead, there is a relative tangential or sliding motion between the crack faces and only a mode II stress intensity factor is generated.

Some experimentalists maintain that mode II crack propagation is impossible and that a crack in a shear field will either be arrested or turn into the normal direction of tensile stress. However, real cracks are never completely plane and Gross[1] has argued that the sliding motion in a slightly curved crack due to shear loading will cause contact between the crack faces. The resulting compressive contact tractions could then cause one or both tips to open, giving a small mode I stress intensity factor, which could exert a significant influence in crack propagation.

In this paper, we examine the possible operation of this mechanism in the idealized case of the sinusoidal crack in an infinite plane.

\section{FORMULATION}

Consider a sinusoidal crack of amplitude $\epsilon$ in the interval of $(-a, a)$ lying on an infinite elastic isotropic plane as shown in Fig. 1. The plane is subjected to a uniform shear stress $\tau$ at infinity, in the direction parallel to the $x$ axis, and the crack remains open throughout its length. In general, we anticipate negative values of crack opening displacement in some regions of the crack and these results will give an indication of the arrangement of contact and separation zones for a physically correct formulation in Section 4 below.

To solve the problem, we use the classical method of representing the perturbation in the local elastic stress field due to the crack by a distribution of dislocation along the crack. The stress components at $(x, y)$ due to a discrete edge dislocation at $(\xi, \eta)$ are [2]

$$
\begin{aligned}
& \sigma_{x x}=-C b_{x}\left\{\frac{y-\eta}{r^{2}}+\frac{2(x-\xi)^{2}(y-\eta)}{r^{4}}\right\}+C b_{y}\left\{-\frac{x-\xi}{r^{2}}+\frac{2(x-\xi)^{3}}{r^{4}}\right\} \\
& \sigma_{x y}=-C b_{x}\left\{\frac{x-\xi}{r^{2}}-\frac{2(x-\xi)^{3}}{r^{4}}\right\}+C b_{x}\left\{-\frac{y-\eta}{r^{2}}+\frac{2(x-\xi)^{2}(y-\eta)}{r^{4}}\right\} \\
& \sigma_{y y}=-C b_{x}\left\{\frac{y-\eta}{r^{2}}-\frac{2(x-\xi)^{2}(y-\eta)}{r^{4}}\right\}+C b_{x}\left\{\frac{3(x-\xi)}{r^{2}}-\frac{2(x-\xi)^{3}}{r^{4}}\right\}
\end{aligned}
$$

where $b_{x}, b_{y}$ are the glide and climb components respectively of the Burgers vector,

$$
\begin{aligned}
& r^{2}=(x-\xi)^{2}+(y-\eta)^{2} \\
& C=\frac{2 \mu}{\pi(\kappa+1)} .
\end{aligned}
$$

$\kappa=3-4 v$ for conditions of plane strain, and $\mu, v$ are the shear modulus and Poisson's ratio respectively for the material. 


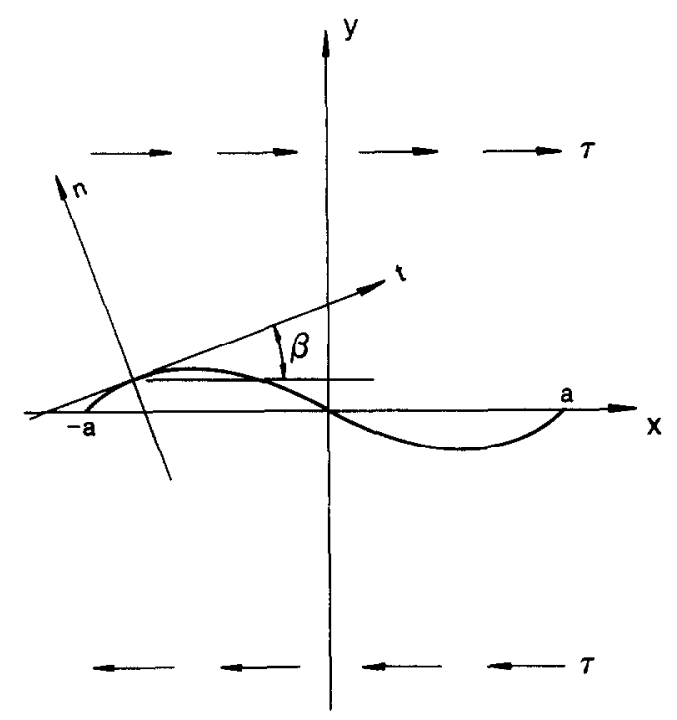

Fig. 1. The sinusoidal crack in shear field.

Since the shape of the crack is defined by $y=-\epsilon \sin \pi x / a$, the normal and the shear stress components on the crack surface are

where

$$
\begin{aligned}
& \sigma_{n}=\sigma_{x x} \sin ^{2} \beta+\sigma_{y y} \cos ^{2} \beta-2 \sigma_{x y} \sin \beta \cos \beta \\
& \sigma_{t}=\left(\sigma_{x x}-\sigma_{y y}\right) \sin \beta \cos \beta-\sigma_{x y}\left(\cos ^{2} \beta-\sin ^{2} \beta\right)
\end{aligned}
$$

$$
\tan \beta=\frac{\mathrm{d} y}{\mathrm{~d} x}=-\pi \frac{\epsilon}{a} \cos \pi x / a
$$

is the local inclination of the crack to the $x$ axis. We define $\gamma=\epsilon / a$ and assume that $\gamma \ll 1$, in which case the expressions $(4,5)$ for the stress components can be simplified.

Taking $\gamma^{2} \approx 0$, in comparison with $\gamma$ and using $y=-\epsilon \sin \pi x / a, \eta=-\epsilon \sin \pi \xi / a$, we have $r^{2} \approx(x-\xi)^{2}, \sin \beta \approx-\gamma \sin \pi x / a$ and $\cos \beta \approx 1$.

Thus, we obtain the stress components on the crack surface at $(x, y)$ due to a dislocation at $(\xi, \eta)$ in the form

$$
\begin{aligned}
\sigma_{n} & =C\left\{\frac{b_{y}}{(x-\xi)}+b_{x}\left(\frac{2 \pi \gamma \cos \pi x / a}{(x-\xi)}-\epsilon \frac{\sin \pi x / a-\sin \pi \eta / a}{(x-\xi)^{2}}\right)\right\} \\
\sigma_{t} & =C\left\{\frac{-b_{x}}{(x-\xi)}+\epsilon b_{y} \frac{\sin \pi x / a-\sin \pi \eta / a}{(x-\xi)^{2}}\right\} .
\end{aligned}
$$

We can now generalize this result by placing a distribution of dislocations of components $B_{x}(x), B_{y}(x)$ along the crack and superposing the undisturbed stress field, $\sigma_{x y}=\tau$, in which case the normal traction $N(x)$ and the shear traction $S(x)$ on the crack surface can be written down as

$$
\begin{gathered}
N(x)=C \int_{-a}^{a}\left\{\frac{B_{y}(\xi)}{x-\xi}+B_{x}(\xi)\left(\frac{2 \pi \gamma \cos \pi x / a}{x-\xi}-\epsilon \frac{\sin \pi x / a-\sin \pi \xi / a}{(x-\xi)^{2}}\right)\right\} \mathrm{d} \xi+2 \pi \gamma \tau \cos \pi x / a ; \\
\quad-a<x<a \\
S(x)=C \int_{-a}^{a}\left\{\frac{-B_{x}(\xi)}{x-\xi}+\epsilon B_{y}(\xi) \frac{\sin \pi x / a-\sin \pi \xi / a}{(x-\xi)^{2}}\right\} \mathrm{d} \xi-\tau ; \quad-a<x<a .
\end{gathered}
$$

We first consider the crack problem under the assumption that the crack remains open. In this case, both traction components must be zero throughout the crack length, leading to the two integral equations

$$
\begin{aligned}
& C \int_{-a}^{a}\left\{\frac{B_{y}(\xi)}{x-\xi}+B_{x}(\xi)\left(\frac{2 \pi \gamma \cos \pi x / a}{x-\xi}-\epsilon \frac{\sin \pi x / a-\sin \pi \xi / a}{(x-\xi)^{2}}\right)\right\} \mathrm{d} \xi+2 \pi \gamma \tau \cos \pi x / a=0 \\
& -a<x<a
\end{aligned}
$$




$$
C \int_{-a}^{a}\left\{\frac{-B_{x}(\xi)}{x-\xi}+\epsilon B_{y}(\xi) \frac{\sin \pi x / a-\sin \pi \xi / a}{(x-\xi)^{2}}\right\} \mathrm{d} \xi-\tau=0 ; \quad-a<x<a .
$$

In addition, we must require that the total Burger vector of the distributed dislocations vanishes, i.e.

$$
\int_{-a}^{a} B_{x}(\xi) \mathrm{d} \xi=0 ; \quad \int_{-a}^{a} B_{y}(\xi) \mathrm{d} \xi=0
$$

to ensure that the displacement is single-valued outside the crack.

It can be shown that the bounded solution for $B_{x}(x)$ and $B_{y}(x)$ will not satisfy the conditions (12). Thus, we anticipate that $B_{x}(x)$ and $B_{y}(x)$ will be singular at both ends of the crack.

\section{NUMERICAL SOLUTION}

Equations (10) and (11) constitute two coupled Cauchy singular integral equations for the unknown functions $B_{x}(x)$ and $B_{y}(x)$. Normalizing the interval by the change of variables

$$
x=a u, \quad \xi=a t
$$

and retaining the same symbols for the functions in the new variables, we obtain

$$
\begin{gathered}
C \int_{-1}^{1}\left\{\frac{B_{y}(t)}{u-t}+\gamma B_{x}(t)\left(\frac{2 \pi \cos \pi u}{u-t}-\frac{\sin \pi u-\sin \pi t}{(u-t)^{2}}\right)\right\} \mathrm{d} t+2 \pi \gamma \tau \cos \pi u=0 \\
C \int_{-1}^{1}\left\{\frac{-B_{x}(t)}{u-t}+\gamma B_{y}(t) \frac{\sin \pi u-\sin \pi t}{(u-t)^{2}}\right\} \mathrm{d} t-\tau=0 \\
\int_{-1}^{1} B_{x}(\xi) \mathrm{d} \xi=0 \\
\int_{-1}^{1} B_{y}(\xi) \mathrm{d} \xi=0 .
\end{gathered}
$$

A numerical solution for these equations can then be found, using the method developed by Erdogan et al.[3]. The characteristic function is of the form

$$
w(x)=\left(1-x^{2}\right)^{-1 / 2} .
$$

We define bounded functions $\phi_{x}, \phi_{y}$ by the relations

$$
B_{x}(x)=C^{-1} \tau w(x) \phi_{x}(x) ; \quad B_{y}(x)=C^{-1} \tau w(x) \phi_{y}(x)
$$

in which case the discretized form of eqs (13-16) becomes

$$
\begin{aligned}
\sum_{i=1}^{n} \frac{\pi}{n} \frac{\phi_{y}\left(t_{i}\right)}{u_{k}-t_{i}}+\gamma \sum_{i=1}^{n} \frac{\pi}{n} \phi_{x}\left(t_{i}\right)\left\{\frac{2 \pi \cos \pi u_{k}}{u_{k}-t_{i}}-\Gamma\left(u_{k}, t_{i}\right)\right\} & =-2 \pi \gamma \cos \pi u_{k} ; \quad k=1,2, \ldots n-1 \\
\sum_{i=1}^{n} \frac{\pi}{n} \frac{\phi_{x}\left(t_{i}\right)}{u_{k}-t_{i}}-\gamma \sum_{i=1}^{n} \frac{\pi}{n} \phi_{y}\left(t_{i}\right) \Gamma\left(u_{k}, t_{i}\right) & =-1 ; k=1,2, \ldots n-1 \\
\sum_{i=1}^{n} \phi_{x}\left(t_{i}\right) & =0 \\
\sum_{i=1}^{n} \phi_{y}\left(t_{i}\right) & =0
\end{aligned}
$$

where

$$
\Gamma\left(u_{k}, t_{i}\right)=\frac{\sin \pi u_{k}-\sin \pi t_{i}}{\left(u_{k}-t_{i}\right)^{2}}
$$


and the choice of the integration and collocation points

$$
t_{i}=\cos \left(\frac{2 i-1}{2 n} \pi\right) ; \quad u_{k}=\cos \left(\frac{k}{n} \pi\right)
$$

is prescribed by the fact that we are seeking the unbounded solution[3].

Equations (19-22) constitute a system of $2 n$ linear equations which can be solved for the $2 n$ unknowns, $\phi_{x}\left(t_{i}\right), \phi_{y}\left(t_{i}\right)$.

The normal displacement between the crack surfaces, i.e. the crack opening displacement $U_{n}(x)$ can be determined from the condition

$$
U_{n}(s)=\cos \beta \int_{-1}^{s} B_{y}(\xi) \mathrm{d} s-\sin \beta \int_{-1}^{s} B_{x}(\xi) \mathrm{d} s .
$$

Since $\gamma \ll 1$, it can be written as

$$
U_{n}(s)=\int_{-1}^{s} B_{y}(\xi) \mathrm{d} s+\pi \gamma \cos \pi s / a \int_{-1}^{s} B_{x}(\xi) \mathrm{d} s .
$$

The integration interval of (23) is not the same as the previous integrals, and hence we cannot use the numerical integration method of Erdogan and Cook[3]. Instead, we calculate the integral by Simpson's method using the values of $B_{x}(x)$ and $B_{y}(x)$ at the integration points. The results show that the relative normal displacement is positive at the crack tips, but negative in the central region. For a crack of initially zero thickness, a negative crack opening displacement is physically meaningless, but this result leads us to anticipate that a contact region will be developed in a central region, the regions near the crack tips remaining open.

\section{THE PARTIALLY CLOSED CRACK}

In view of the results of Section 3, we now reconsider the problem under the assumption that a central region of the crack, $|x|<c$, remains closed, as shown in Fig. 2. For generality, we also add normal tractions $\sigma$ at infinity, but we are mostly interested in effect of the shear tractions.

In this case, the crack opening displacement, $U_{n}(x)=0$ throughout the closed region, leading to

$$
\int_{-a}^{x} B_{y}(s) \mathrm{d} s+\pi \gamma \cos \pi x / a \int_{-a}^{x} B_{x}(s) \mathrm{d} s=0 ; \quad-c<x<c
$$

from eq. (23).

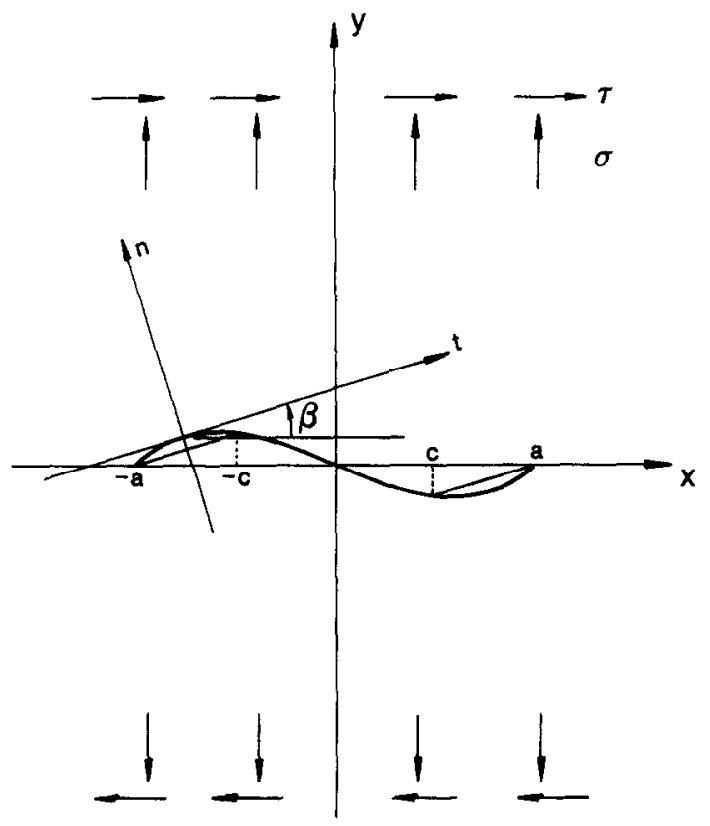

Fig. 2. The sinusoidal crack with central contact zone. 
Also, the open regions of the crack must be traction free and, in particular, $N(x)=0$, giving $C \int_{-a}^{a}\left\{\frac{B_{y}(s)}{x-s}+B_{x}(s)\left(\frac{2 \pi y \cos \pi x / a}{x-s}-\epsilon \frac{\sin \pi x / a-\sin \pi s / a}{(x-s)^{2}}\right)\right\} \mathrm{d} s$

$$
+2 \pi \gamma \tau \cos \pi x / a+\sigma=0 ; \quad c<|x|<a .
$$

We assume that the contact is frictionless and hence the shear traction $S(x)=0$ throughout the crack, i.e.

$$
C \int_{-a}^{a}\left\{\frac{-B_{x}(s)}{x-s}+\epsilon B_{y}(s) \frac{\sin \pi x / a-\sin \pi s / a}{(x-s)^{2}}\right\} \mathrm{d} s-\tau+\pi \gamma \sigma \cos \pi x / a=0 ; \quad-a<x<a .
$$

The parameter, $c$, defining the extent of the contact zone is initially unknown and must be found as part of the solution from the unilateral contact inequalities

$$
\begin{gathered}
U_{n}(x)=\int_{-a}^{x} B_{y}(s) \mathrm{d} s+\pi \gamma \cos \pi x / a \int_{-a}^{x} B_{x}(s) \mathrm{d} s \geqslant 0 ; \quad c<|x|<a \\
N(x)=C \int_{-a}^{a}\left\{\frac{B_{y}(s)}{x-s}+B_{x}(s)\left(\frac{2 \pi \gamma \cos \pi x / a}{x-s}-\epsilon \frac{\sin \pi x / a-\sin \pi s / a}{(x-s)^{2}}\right)\right\} \mathrm{d} s \\
+2 \pi \gamma \tau \cos \pi x / a+\sigma \leqslant 0 ; \quad|x|<c
\end{gathered}
$$

which states that interpenetration of the crack and tensile contact tractions respectively are physically inadmissible. They are formally equivalent to the condition that the contact traction should tend to zero rather than be unbounded as $x \rightarrow \pm c$.

Finally, the closure conditions (12) must also be imposed, implying an unbounded solution for $B_{x}, B_{y}$ as $x \rightarrow \pm a$.

Normalizing in the interval $(-a,+a)$ and introducing the same characteristic function $w(x)$, the problem becomes

$$
\begin{aligned}
& \int_{-1}^{t} w(t)\left\{\frac{\phi_{y}(t)}{u-t}+\gamma \phi_{x}(t)\left(\frac{2 \pi \cos \pi u}{u-t}-\frac{\sin \pi u-\sin \pi t}{(u-t)^{2}}\right)\right\} \mathrm{d} t+2 \pi \gamma \cos \pi u+\delta=0 ; \\
& \alpha<|u|<1 \\
& \int_{-1}^{1} w(t)\left\{\frac{-\phi_{x}(t)}{u-t}+\gamma \phi_{y}(t) \frac{\sin \pi u-\sin \pi t}{(u-t)^{2}}\right\} \mathrm{d} t-1+\pi \gamma \delta \cos \pi u=0 ; \quad-1<u<1 \\
& \int_{-1}^{1} w(t)\left\{\frac{\phi_{y}(t)}{u-t}+\gamma \phi_{x}(t)\left(\frac{2 \pi \cos \pi u}{u-t}-\frac{\sin \pi u-\sin \pi t}{(u-t)^{2}}\right)\right\} \mathrm{d} t+2 \pi \gamma \cos \pi u+\delta \leqslant 0 \\
& -\alpha<u<\alpha \\
& \int_{-1}^{u} w(t) \phi_{y}(t) \mathrm{d} t+\pi \epsilon \cos \pi u \int_{-1}^{u} w(t) \phi_{x}(t) \mathrm{d} t=0 ; \quad-\alpha<u<\alpha \\
& \int_{-1}^{u} w(t) \phi_{y}(t) \mathrm{d} t+\pi \epsilon \cos \pi u \int_{-1}^{u} w(t) \phi_{x}(t) \mathrm{d} t \geqslant 0 ; \quad \alpha<|u|<1 \\
& \int_{-1}^{1} w(t) \phi_{x}(t) \mathrm{d} t=0 \\
& \int_{-1}^{1} w(t) \phi_{y}(t) \mathrm{d} t=0
\end{aligned}
$$

where $\alpha=c / a$ and $\delta=\sigma / \tau$.

The problem is non-linear in consequence of the inequality conditions $(27,28)$ and hence must be solved by an iterative method. Assume that

$$
c=\left|\cos \frac{K+1}{n} \pi\right|
$$


The discretized form of conditions $(29-35)$ is:

$$
\begin{aligned}
& \sum_{i=1}^{n} \frac{\pi}{n} \frac{\phi_{u}\left(t_{i}\right)}{u_{k}-t_{i}}+\gamma \sum_{i=1}^{n} \frac{\pi}{n} \phi_{x}\left(t_{i}\right)\left\{\frac{2 \pi \cos \pi u_{k}}{u_{k}-t_{i}}-\Gamma\left(u_{k}, t_{i}\right)\right\}=-2 \pi \gamma \cos \pi u_{k}-\delta ; \\
& k=1,2, \ldots, K ; n-K, n-K+1, \ldots n-1 \\
& \sum_{i=1}^{n} \frac{\pi}{n} \frac{\phi_{x}\left(t_{i}\right)}{u_{k}-t_{i}}-\gamma \sum_{i=1}^{n} \frac{\pi}{n} \phi_{y}\left(t_{i}\right) \Gamma\left(u_{k}, t_{i}\right)=-1-\pi \gamma \cos \pi u_{k} ; \quad k=1,2, \ldots n-1 \\
& \sum_{i=1}^{n} \frac{\pi}{n} \frac{\phi_{y}\left(t_{i}\right)}{u_{k}-t_{i}}+\gamma \sum_{i=1}^{n} \frac{\pi}{n} \phi_{x}\left(t_{i}\right)\left\{\frac{2 \pi \cos \pi u_{k}}{u_{k}-t_{i}}-\Gamma\left(u_{k}, t_{i}\right)\right\} \leqslant-2 \pi \gamma \cos \pi u_{k}-\delta ; \\
& k=K+1, K+2, \ldots n-K-2, n-K-1 \\
& f_{y 1}+\frac{1}{2} \sum_{i=1}^{K-1}\left(B_{y}\left(t_{i}\right)+B_{y}\left(t_{i+1}\right)\left(t_{i+1}-t_{i}\right)+f_{y K}\right. \\
& +\pi \epsilon \cos \pi u_{k}\left\{f_{x i}+\frac{1}{2} \sum_{i=1}^{K-1}\left(B_{x}\left(t_{i}\right)+B_{x}\left(t_{i+1}\right)\left(t_{i+1}-t_{i}\right)+f_{x K}\right\}=0 ;\right. \\
& k=K+1, K+2, \ldots n-K-2, n-K-1 \\
& f_{y 1}+\frac{1}{2} \sum_{i=1}^{K-1}\left(B_{y}\left(t_{i}\right)+B_{y}\left(t_{i+1}\right)\left(t_{i+1}-t_{i}\right)+f_{y K}\right. \\
& +\pi \epsilon \cos \pi u_{k}\left\{f_{x 1}+\frac{1}{2} \sum_{i=1}^{K-1}\left(B_{x}\left(t_{i}\right)+B_{x}\left(t_{i+1}\right)\left(t_{i+1}-t_{i}\right)+f_{x K}\right\} \geqslant 0 ;\right. \\
& k=1,2, \ldots, K ; n-K, n-K+1, \ldots n-1 \\
& \sum_{i=1}^{n} \phi_{y}\left(t_{i}\right)=0 \\
& \phi_{x}\left(t_{i}\right)=0
\end{aligned}
$$

where

$$
\begin{aligned}
\Gamma\left(u_{k}, t_{i}\right) & =\frac{\sin \pi u_{k}-\sin \pi t_{i}}{\left(u_{k}-t_{i}\right)^{2}} \\
f_{r 1} & =-2 B_{r}\left(t_{1}\right)\left(1+t_{1}\right) ; \quad r=x, y \\
f_{r K} & =\frac{1}{2}\left\{2 B_{r}\left(t_{K}\right)+\frac{B_{r}\left(t_{K+1}\right)-B_{r}\left(t_{K}\right)}{t_{K+1}-t_{K}}\right\}\left(u_{K}-t_{K}\right) ; \quad r=x, y \\
B_{r}\left(t_{i}\right) & =w\left(t_{i}\right) \phi_{r}\left(t_{i}\right)
\end{aligned}
$$

and the choice of the integration and collocation points

$$
t_{i}=\cos \left(\frac{2 i-1}{2 n} \pi\right) ; \quad u_{k}=\cos \left(\frac{k}{n} \pi\right)
$$

is prescribed by the fact that we are seeking the unbounded solution[3] in this case as well.

The results for the open crack are used to obtain an initial guess for $c$ after which iteration proceeds by changing the contact status (i.e. $K$ ) of collocation points for which the appropriate inequality is violated. Notice however that the accuracy of the final value of $c$ is restricted by the finite spacing of the collocation points.

\section{RESULTS}

The problem is solved for different values of $\delta$ with $n=100$ and $\gamma=0.001$. From the calculations, we find that the contact status of the crack $h$ varies with $\delta$, i.e. the ratio of normal stress to shear stress. There are some special values of $\delta: \delta_{1}=0.00099065 ; \delta_{2}=-0.00335058$; $\delta_{3}=-0.00368505$. 


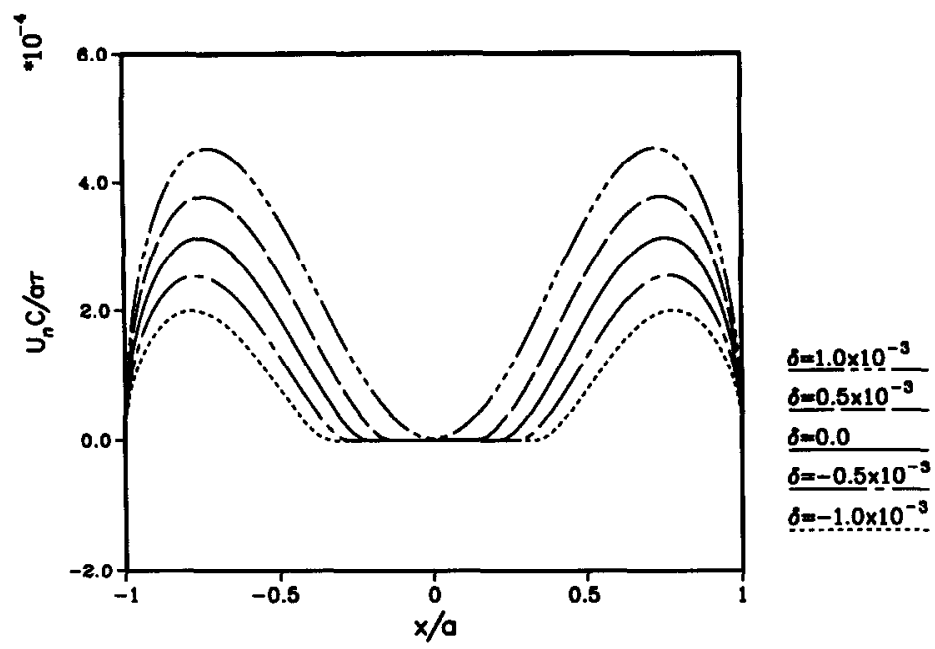

Fig. 3. Gap profiles for various values of $\sigma / \tau$.

For $\delta$ greater than $\delta_{1}$, the crack will be fully open; while for $\delta$ smaller than $\delta_{3}$, it will be completely closed; for $\delta$ between $\delta_{2}$ and $\delta_{1}$ there is central contact zone, but for a small range of $\delta$ between $\delta_{3}$ and $\delta_{2}$, there are three contact zones: one at the center and two at the crack tips.

Extensive study was done for the case of $\delta$ between $\delta_{2}$ and $\delta_{1}$. Some of the results are shown in Figs 3 and 4, which feature the case of the central contact zone.

The stress intensity factors $K_{\mathrm{I} . I I}$ for the tensile and shear stresses respectively at $x=-a$ are defined as

$$
K_{\mathrm{I}, \mathrm{II}}=\lim _{x \rightarrow-a} \sqrt{2 \pi(x+a)}\left[\sigma_{n}, \sigma_{t}(x, 0)\right]
$$

and can be related to the dislocation distributions by the relations

$$
K_{1, I I}=\frac{2 \mu \sqrt{\pi / a}}{k+1} \lim _{s \rightarrow-1}\{2(s+1)\}^{-1 / 2}[g(s), h(s)]
$$

where

$$
\begin{gathered}
g(s)=U_{n}(s), \\
h(s)=\int_{-1}^{s} B_{x}(\xi) \mathrm{d} \xi-\pi \gamma \cos \pi s \int_{-1}^{s} B_{y}(\xi) \mathrm{d} \xi
\end{gathered}
$$

are the relative normal and tangential displacements respectively.

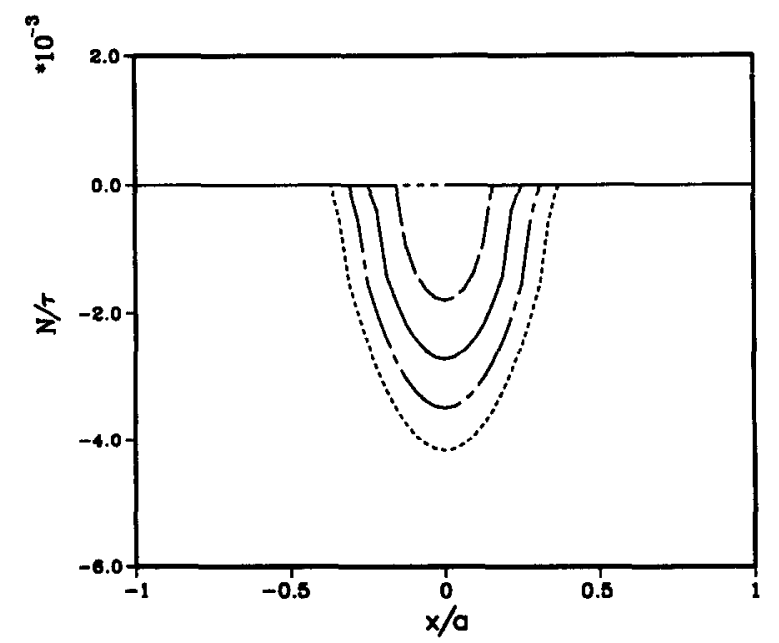

Fig. 4. Contact pressure for various values of $\sigma / \tau$. 


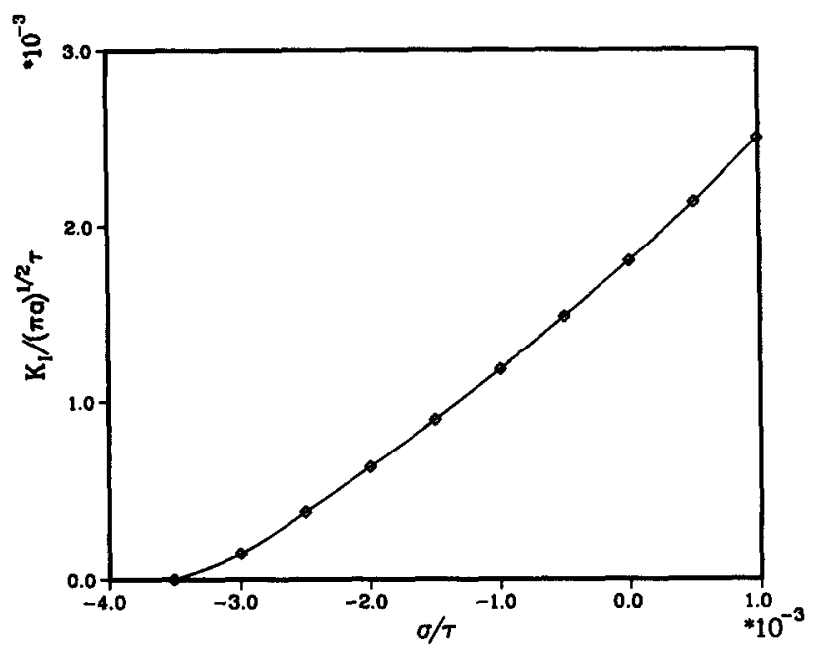

Fig. 5. Normalized mode I stress intensity factor vs $\sigma / \tau$.

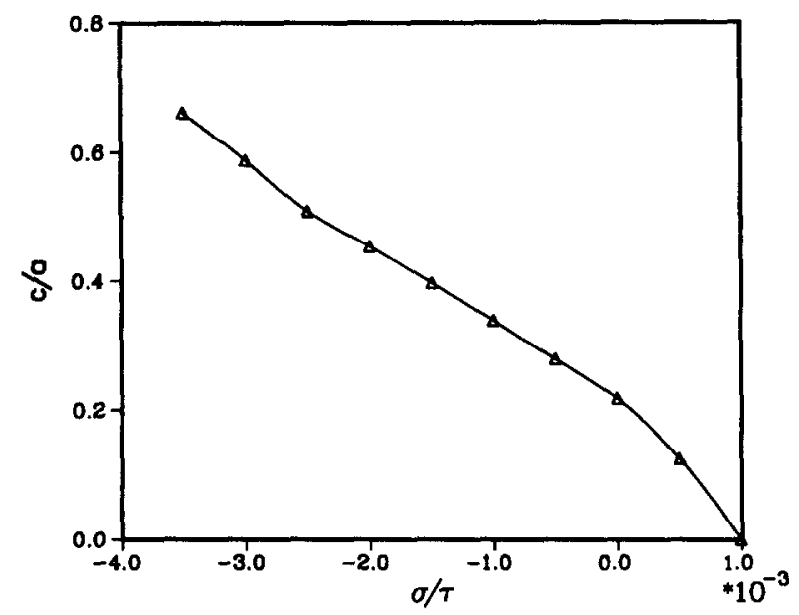

Fig. 6. Extent of contact vs $\sigma / \tau$.

After some mathematical manipulation, $K_{\mathrm{l}}$ and $K_{\mathrm{ll}}$ are obtained as

$$
\begin{aligned}
& K_{\mathrm{I}}=\pi \tau \sqrt{\pi a}\left\{\phi_{y}(-1)-\pi \gamma \phi_{x}(-1)\right\} \\
& K_{\mathrm{II}}=\pi \tau \sqrt{\pi a}\left\{\phi_{x}(-1)+\pi \gamma \phi_{y}(-1)\right\} .
\end{aligned}
$$

When calculating $K_{1}$ and $K_{11}, \phi_{x}(-1)$ and $\phi_{y}(-1)$ are obtained by linear interpolation of $\phi_{x}$ and $\phi_{y}$ at the nearest two integration points.

From Figs 5 and 6, we can see clearly that $K_{1}$ varies approximately linearly with the ratio of the normal stress to shear stress and the contact ratio declines, but $K_{1 \mathrm{I}} / \sqrt{\pi a} \tau$ remains practically equal to 1 .

Convergence of the method was spot-checked for $K_{1}$ and $K_{\mathrm{II}}$ using 50,100 and 200 intermediate data points. This check indicated a difference of less than $10^{-5}$ and therefore $n=100$ was chosen for the calculation.

\section{REFERENCES}

[1] T. S. Gross, Frictional effects in mode III fatigue crack propagation. Scripta Metall. 19, 1185-1188 (1985).

[2] J. Dundurs, Elastic interaction of dislocations with inhomogeneities, in Mathematical Theory of Dislocations (Edited by T. Mura). The American Society of Mechanical Engineers (1969).

[3] F. Erdogan, G. D. Gupta and T. S. Cook, Numerical solution of singular integral equations, in Methods of Analysis and Solutions of Crack Problems (Edited by G. C. Sih). Noordhoff, Leyden (1973). 